\title{
A Time-Slot Routing Protocol for Smart Home
}

\author{
Xiao Tian, Nan Chen, Xian-Deng He \\ School of Telecommunications Engineering, Xidian University, Xi' an, China \\ E-mail: t_xiaodouzi@163.com,nchen@mail.xidian.edu.cn, xdhe@mail.xidian.edu.cn
}

\begin{abstract}
By adopting ad hoc technique, the routing protocol of data network can be realized in smart home system. Due to the features of low moving speed, less number of nodes and high data rate, few present ad-hoc protocols are suitable for smart home data network. In order to make up the deficiencies of present protocols, and solve the network congestion problem, a time-slot routing protocol is proposed in this paper. According to different nodes, the optimum number of slots is analyzed and given, which will provide a theoretical basis for practice.
\end{abstract}

Keywords- ad hoc; smart home; time slot

\section{INTRODUCTION}

By combining the technologies of network communications, automatic control, security and generic cabling, the smart home system provides people an intelligent, comfortable and convenient life [1]. There are two kinds of networks in smart home: the control network and the data network. The control network is mainly used for the operations of electrical household appliances, while the data network is for high speed data interconnection among appliances [2]. A structure of smart home is shown in Figure 1 .

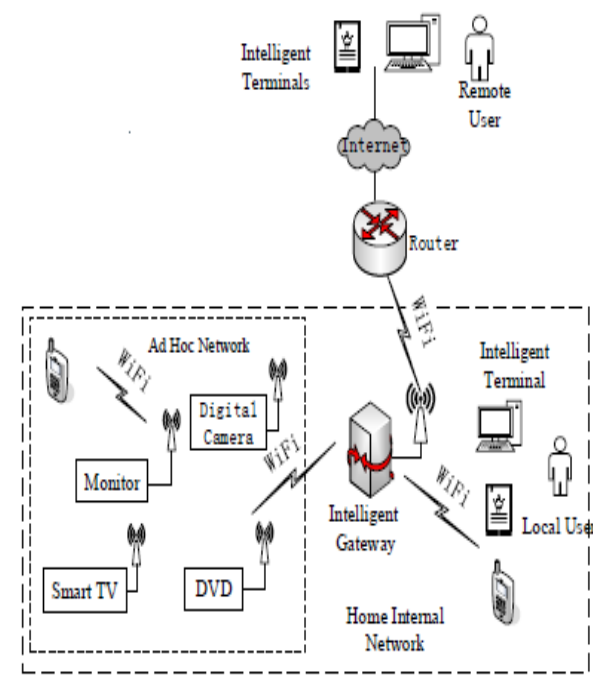

Figure 1. The structure of smart home system.

The wireless transmission in Figure 1 is based on WiFi, which is an ideal choice for family networks. By making use of the ad hoc and WiFi techniques, higher quality of signal and service can be provided for users [3-6]. So with the ad hoc and WiFi techniques, a high-speed data network and a larger wireless coverage will be provided for users in the smart home system.
There are many routing protocols proposed for ad hoc networks. [7] mentions a location-based directed diffusion routing protocol. In [8], an optimum wireless sensor network routing protocol has been introduced. A ZigBee-based routing protocol using UPPAAL (a tool suit for automatic verification of real-time systems) has been introduced in [9]. A multi metric on-demand ad-hoc routing protocol has been given in [10]. RPL (Routing Protocol for LLN) based multipath routing protocols have been proposed in [11]. These protocols can be applied to some network with large number of nodes, low data rate and high moving speed, but not suitable for the smart home data network, which has the features of less number of nodes, high data rate and low moving speed.

A time-slot routing protocol (TSRP) of smart home data network has been proposed in this paper. In this protocol, the process of routing discovery is started by the gateway, and considering the scale of a home, the nodes are divided into three levels according to the received signal strength. The congestion problem in the network layer can be eased and the routing stability can be enhanced by the application of the time-slot technique. Considering the influence of packet loss rate and delay on the network, a routing selection method, based on immune particle swarm optimization algorithm, is used in this paper [12]. In order to reduce the average network access time, curves of the optimum slots' number with the net nodes' number are given in this paper, which will gives a theoretical basis for practice.

Compared to using relays or multi-gateway in home to get a larger wireless coverage, the proposed protocol, which adopts Ad hoc technique and dynamic routing discovery technique, makes the internet connection more movable and flexible. Using just one intelligent gateway, the proposed protocol provides a bridge between internal and external network, as well as a much safer smart home network. Besides, via the intelligent gateway, external network can control all internal devices, and the internal devices can report its running status to external networks. In addition, local smart terminals can connect to the intelligent gateway directly even without the internet connection, and can control all kinds of household appliances and devices.

\section{DESIGN OF ROUTING PROTOCOLS}

In this paper, considering the received signal strength and the area of a house, nodes can be divided into three levels: the first-level nodes, the second-level nodes and the thirdlevel nodes. The first-level nodes can access the gateway directly, the second-level nodes can access the gateway with two hops, and the third-level nodes can access the gateway 
with three hops. The gateway can access the Internet, and controlled by the gateway, these nodes can access the Internet in order. In smart home system, the most common demand is the nodes accessing to the Internet, which is this paper discussed.

\section{A. Discovery of the first-level nodes}

When the gateway broadcasts allow_1 packet to the whole network, all the nodes in its first hop range receive the packet almost at the same time. In order to avoid the collisions when nodes send requests to the gateway, a method of binary time-slot division and adaptive back-off algorithm is used in this protocol. Besides, the maximum retransmission times is set to 4 .

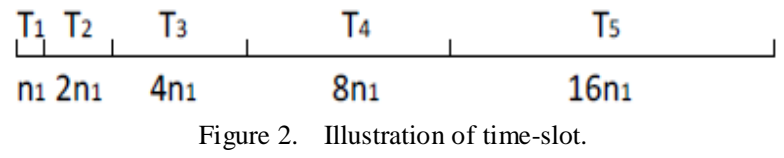

In Figure 2, there are five segments with 4 times retransmission, $\mathrm{T}_{1}=\mathrm{T}, \mathrm{T}_{2}=2 \mathrm{~T}, \mathrm{~T}_{3}=4 \mathrm{~T}, \mathrm{~T}_{4}=8 \mathrm{~T}, \mathrm{~T}_{5}=16 \mathrm{~T}$, and $\mathrm{T}=\mathrm{n}_{1} \tau$, where $\tau$ is the duration of a time-slot. Firstly, in segment $T_{1}$, the first-level node randomly selects one from $n_{1}$ time-slots to send request packet. If the node does not receive the rreq_ack packet send from the gateway within this time-slot, it will select one from $2 \mathrm{n}_{1}$ time-slots to send request packet again in segment $T_{2}$, and so on. If the node still does not receive the rreq_ack packet after 4 transmissions, it has to wait for the next allow_1 packet from the gateway. If the gateway receives the request packet sent from the node successfully, it will send reply packet, and then the route between the gateway and the first-level nodes are saved to the routing tables.

The HELLO packet and the RRER packet, which are used for route maintenance, are the same as those in AODV (Ad hoc On-Demand Distance Vector Routing) [13], and they will not be discussed in this paper. To avoid receiving the same packet, the approach of serial number is also adopted as AODV. There are only three elements in the routing table of each node, which are the destination address, the next hop address and the level this node belongs to.

\section{B. Discovery of the second-level nodes}

Having accessed the Internet, the first-level node will receive an allow_2 packet from gateway respectively according to the routing table. Then it will broadcast this packet to all its neighborhood nodes in a special time-slot designated by the gateway. It is assumed that the time for sending any packet is one time-slot, and the number of firstlevel nodes is $m_{1}$, so the broadcast of allow_2 packets will cost a time of $m_{1} \tau$. And the retransmission order of allow_2 packet is distributed by the gateway during Section II. $\bar{A}$. When the neighborhood nodes receive allow_2 packets, they will check their routing tables whether there is a routing path to the gateway. If not, they will mark themselves to be the second-level nodes, and send the request packet to the firstlevel node. Same as the first-level nodes do, they also takes the method of binary exponential backoff algorithm with similar parameters. In this section, each $\mathrm{T}$ has $\mathrm{n}_{2}$ time-slots.
As each second-level node may receive allow_2 packet from more than one first-level nodes, there will be $m_{2}^{*}=\mathrm{m}_{2} \times \mathrm{m}_{1} \times \mathrm{P}_{\mathrm{r} 1}$ nodes compete to send RREQ packets. Here, $\mathrm{m} 2$ is the number of second-level nodes and $\operatorname{Pr}_{1}$ is the repeat probability. When the first-level nodes receive RREQ packets from the second-level nodes, they will forward these packets to the gateway at once. Then the gateway will choose one routing path according to the method introduced in [12] to send reply packet, and then the routes between the gateway and the second-level nodes are saved to the routing tables.

\section{Discovery of the third-level nodes}

Having accessed the Internet, the second-level node will receive an allow_3 packet from the gateway respectively according to the routing table. Then it will broadcast this packet to all its neighborhood nodes in a similar way like Section II.B. And there will be $m_{3}^{*}=m_{3} \times m_{2} \times \mathrm{P}_{\mathrm{r} 2}$ nodes compete to send RREQ packets, in which $m_{3}$ is the number of third-level nodes and $\mathrm{Pr}_{2}$ is the repeat probability. Each $\mathrm{T}$ has $\mathrm{n}_{3}$ time-slots. When the second-level nodes receive RREQ packets from the third-level nodes, they will forward these packets to the gateway at once. Then the gateway will choose one routing path according to the method introduced in [12] to send reply packet. At this time, the routes between the gateway and the third-level nodes are saved to the routing tables.

\section{ANALYSIS AND SIMULATION}

\section{A. System model}

In this section, we will analyze the average network access time, which is affected by the processing of sending RREQ packets. The success probabilities of sending RREQ packet in segments $T_{1}, T_{2}, T_{3}, T_{4}$ and $T_{5}$, are presented by $P_{1}$, $\mathrm{P}_{2}, \mathrm{P}_{3}, \mathrm{P}_{4}$ and $\mathrm{P}_{5}$. According to the deduction in Appendix A, $\mathrm{P}_{\mathrm{j}}$ can be given by

$$
P_{j}=\left(\frac{2^{j-1} n_{1}-1}{2^{j-1} n_{l}}\right)^{\left[\prod_{i=I}^{j-l}\left(1-P_{i}\right) m_{l}\right]-1}, j=1,2,3,4,5
$$

In which, “ $\lceil x\rceil$ ” means the rounded up of $\mathrm{x}$.

The average time it takes for a node to send RREQ packet is shown in TABLE I.

TABLE I. PROBABILITY DISTRIBUTION AND AVERAGE TIME

\begin{tabular}{|c|c|c|c|c|}
\hline $\begin{array}{c}\text { segment to } \\
\text { be } \\
\text { discovered }\end{array}$ & $T 1$ & $T 2$ & $\cdots$ & $T 5$ \\
\hline $\begin{array}{c}\text { Probability } \\
\text { to be } \\
\text { discovered }\end{array}$ & $P_{1}^{*}=P_{1}$ & $P_{2}^{*}=\left(1-P_{1}\right) P_{2}$ & $\cdots$ & $P_{5}^{*}=\prod_{i=1}^{4}\left(1-P_{i}\right) P_{5}$ \\
\hline $\begin{array}{c}\text { Average } \\
\text { time }\end{array}$ & $T_{1}^{*}=T_{1} / 2$ & $T_{2}^{*}=T_{1}+T_{2} / 2$ & & $T_{5}^{*}=\sum_{i=1}^{4} T_{i}+T_{5} / 2$ \\
\hline
\end{tabular}




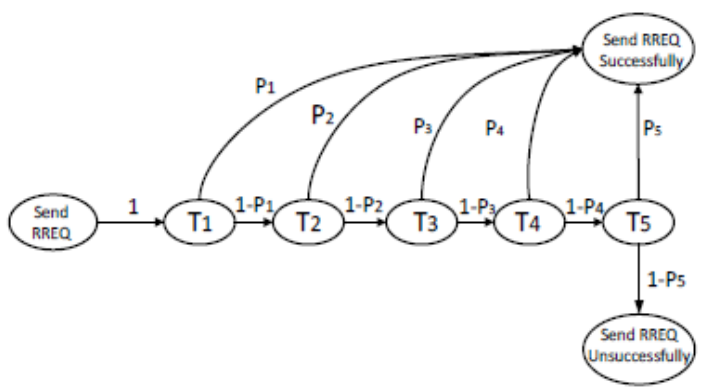

Figure 3. The diagram of transition probability.

Due to the existence of packet loss rate, the success probability of route discovery is hardly to be $100 \%$. In fact, a number greater than Plimit is often adopted. So the average time it takes to send RREQ packet successfully can be presented by segmented function

$$
\bar{T}=\sum_{i=1}^{k} T_{i}^{*} P_{i}^{*}, \text { if } \sum_{i=1}^{k} P_{i}^{*}>P_{\text {limit }}, k=1,2,3,4,5
$$

where $\mathrm{k}$ is the period when nodes to be discoveried, and the average network access time of the first-level nodes can be represented by

$$
\overline{T_{1}} \approx \bar{T}+m_{1} T_{s} P_{\text {limit }}
$$

where Ts is the time for routing selection, which is determined by the routing algorithm.

Because the discovered processes of the second-level and third-level nodes are similar to that of the first-level nodes, the average network access time for the second-level and third-level nodes can be given as (4) and (5).

$$
\begin{gathered}
\overline{T_{2}} \approx T_{1 \max }+\mathrm{m}_{1} \tau+\left.\bar{T}\right|_{n_{1}=n_{2}, m_{1}=m_{2}{ }^{*}} \\
\overline{T_{3}} \approx T_{1 \max }+T_{2 \max }+m_{2}{ }^{*} \tau+\left.\bar{T}\right|_{n_{1}=n_{3}, m_{1}=m_{3}{ }^{*}}
\end{gathered}
$$

where $T_{1 \max }$ and $T_{2 \max }$ are the maximum network access time for the first-level and second-level nodes. $\mathrm{T}_{1 \max }$ and $\mathrm{T}_{2 \max }$ can be given by (6) and (7).

$$
\begin{aligned}
T_{I \max } & =\sum_{i=1}^{k_{1}}\left(2^{i-1} n_{1}\right)+m_{l} T_{s} P_{\text {limit }}, \\
& \text { if } \sum_{i=1}^{k_{1}} P_{i}^{* *}>P_{\text {limit }}, k_{1}=1,2,3,4,5
\end{aligned}
$$

where $\mathrm{k} 1$ is the period when the first-level nodes to be discoveried.

$$
\begin{aligned}
T_{2 \max } & =\sum_{i=1}^{k_{2}}\left(2^{i-1} \mathbf{n}_{2}\right)+m_{2}^{*} T_{s} P_{\text {limit }}, \\
& \text { if } \sum_{i=1}^{k_{2}} P_{i}^{*}>P_{\text {limit }}, k_{2}=1,2,3,4,5
\end{aligned}
$$

where $\mathrm{k}_{2}$ is the period when the second-level nodes to be discoveried.

In conclusion, the average network access time for all nodes is

$$
T=\frac{m_{1} \overline{T_{1}}+\mathrm{m}_{2} \overline{T_{2}}+\mathrm{m}_{3} \overline{T_{3}}}{m_{1}+m_{2}+m_{3}}
$$

\section{B. Simulation and performance analysis}

Considering the data rate of $\mathrm{WiFi}$ and the size of packet, it is assumed that $\tau=1 \mathrm{~ms}, \mathrm{Ts}=20 \tau$, Plimit $=98 \%$ and all nodes can be discovered in 4 times transmission. The relationships between the average access time and the number of slots are given by MATLAB simulation. Figure 4 to Figure 6 are the performance of the first-level nodes.

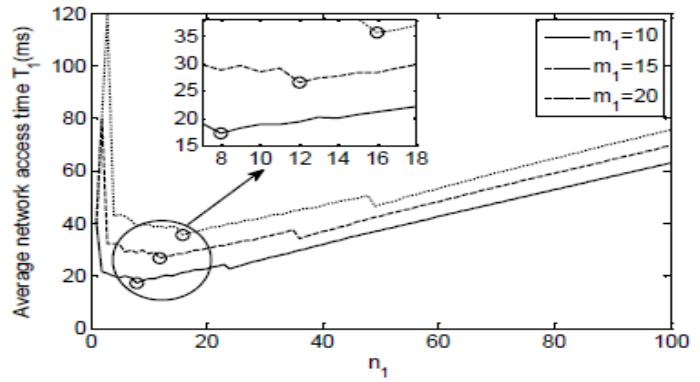

Figure 4. The curves of the average network access time with the number of slots.

Figure 4 shows the curves of the average network access time with the number of slots. It can be seen from the Figure 4 that when the number of first-level nodes is given, the optimum number of slots exists to make average network access time to be shortest, and the number of slots should be set to 8,12 and 16 , when there are 10,15 and 20 first-level nodes, respectively.

Through the calculation of different numbers of the firstlevel nodes, a useful conclusion is shown in Figure 5.

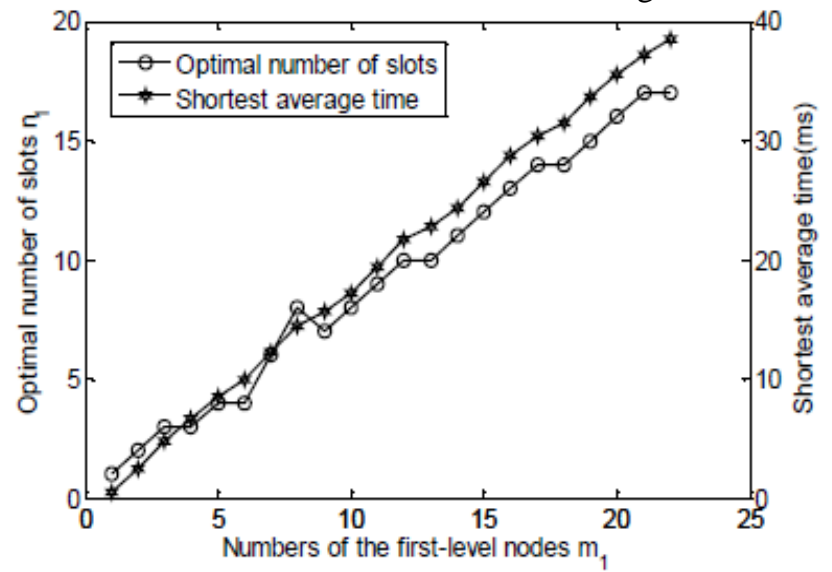

Figure 5. The curves of optimum numbers of slots and its shortest average network access time with the number of nodes.

As shown in Figure 5, the curves give the optimum numbers of slots under different nodes in one level. In a real 
smart home system, the number of the first-level nodes can be estimated according to the distance and surroundings, or can be got from the latest routing discovery, so the proper number of slots can be set to reach the shortest average access time, which is also shown in Figure 5.

From (6) and (7), the curve of maximum network access time with the number of slots can be obtained, which is shown in Figure 6. If $\mathrm{ml}$ is the number of nodes, then it will get $\mathrm{T}_{1 \max }$, and if $\mathrm{m}_{2} *$ is the number of nodes, then it will get $\mathrm{T}_{2 \max }$. As shown in Figure 6 , when the numbers of nodes are 5,10 and 15, the maximum network access times are $126 \mathrm{~ms}$, $252 \mathrm{~ms}$ and $378 \mathrm{~ms}$, respectively.

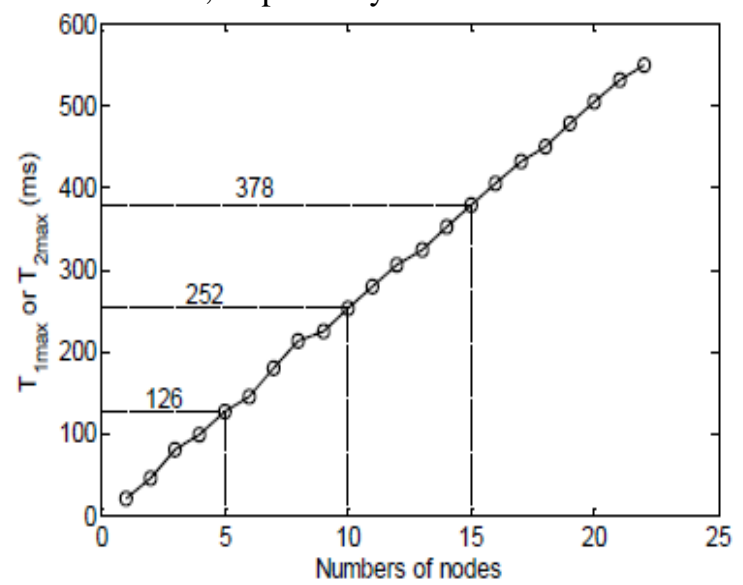

Figure 6. The curve of maximum network access time with the number of slots.

In order to make use of the conclusions from this paper, following steps could be used:

Step 1: estimating the parameters of the system, for example, $\mathrm{m}_{1}=10, \quad \mathrm{~m}_{2}=5, \quad \mathrm{~m}_{3}=5, \quad \tau=1 \mathrm{~ms}, \quad \mathrm{~T}_{\mathrm{s}}=20 \tau, \quad \mathrm{P}_{\text {limit }}=98 \%$, $\operatorname{Pr}_{1}=\operatorname{Pr}_{2}=20 \%$.

Step 2: calculating $m_{2} *=10 \times 5 \times 20 \%=10, m_{3}^{*}=5 \times 5 \times$

$20 \%=5$.

Step 3: finding the optimal numbers of slots from Figure 5, which are $n_{1}=8, n_{2}=8$ and $n_{3}=4$.

Step 4: finding $\mathrm{T}_{1 \max }$ and $\mathrm{T}_{2 \max }$ with the help of Figure 6 , and getting $T_{1 \max }=252 \mathrm{~ms}, T_{2 \max }=252 \mathrm{~ms}$.

Step 5: calculating $\overline{T_{1}}, \overline{T_{2}}, \overline{T_{3}}$ and with the help of (3), (4) and (5), and getting $\bar{T}_{1} \approx 213.205 \mathrm{~ms}, \bar{T}_{2} \approx 279.205 \mathrm{~ms}$, $\overline{T_{3}} \approx 522.598 \mathrm{~ms}$.

Step 6: using (8), the optimal average access time for all nodes is given by $360.3545 \mathrm{~ms}$.

\section{CONCLUSION}

In order to set up a smart home data network with the features of low moving rate, less number of nodes, and high data rate, a time-slot routing protocol is proposed in this paper. After modeling and simulation, the optimal number of slots and its average network access time for different number of nodes are given. Finally, one example of how to using the result in a real smart home system is given.

\section{APPENDIX A---DERIVATION OF $P j$}

It is assumed that there are $\mathrm{ml}$ first-level nodes in the smart home system and $n 1$ time-slots. The probabilities of sending RREQ packet successfully in period $\mathrm{T}_{1}, \mathrm{~T}_{2}, \mathrm{~T}_{3}, \mathrm{~T}_{4}$ and $\mathrm{T}_{5}$, can be presented by $\mathrm{P}_{1}, \mathrm{P}_{2}, \mathrm{P}_{3}, \mathrm{P}_{4}$ and $\mathrm{P}_{5}$. According to [14], the probability $\mathrm{P} 1$ can be given by

$$
\mathrm{P}_{1}=\frac{n_{1}\left(n_{1}-1\right)^{\left(m_{1}-1\right)}}{n_{1}^{m_{1}}}=\left(\frac{n_{1}-1}{n_{1}}\right)^{m_{1}-1}
$$

The nodes that sent RREQ packet unsuccessfully in period $\mathrm{T}_{1}$ will randomly choose one from $2 \mathrm{n}$ slots in period $\mathrm{T}_{2}$ to send request again. The number of nodes that have to send request again in $\mathrm{T}_{2}$ is $\left\lceil\left(1-P_{1}\right) \mathrm{n}_{1}\right\rceil$, where “ “ \lceil\rceil , means the smallest integer larger than $\mathrm{x}$. So the probability $\mathrm{P}_{2}$ is

$$
\mathbf{P}_{2}=\left(\frac{2 n_{1}-1}{2 n_{1}}\right)^{\left\lceil\left(1-P_{1}\right) m_{1}\right\rceil-1}
$$

Similar to $\mathrm{T}_{1}, \mathrm{~T}_{2}$, the probabilities of sending RREQ packet successfully in period $T_{3}, T_{4}$ and $T_{5}$ are

$$
\left.\mathrm{P}_{\mathrm{j}}=\left(\frac{2^{\mathrm{j}-1} \mathrm{n}_{1}-1}{2^{\mathrm{j}-1} \mathrm{n}_{1}}\right)^{\left[\prod_{\mathrm{i}=1}^{\mathrm{j}-1}\left(1-\mathrm{P}_{\mathrm{i}}\right) m_{1}\right.}\right]^{-1}, \mathrm{j}=3,4,5
$$

In conclusion, ${ }_{j}$ can be given by

$$
P_{j}=\left(\frac{2^{j-1} n_{1}-1}{2^{j-1} n_{1}}\right)^{\left[\prod_{i=1}^{j-1}\left(1-P_{i}\right) m_{1}\right]-1}, j=1,2,3,4,5
$$

\section{ACKNOWLEDGMENT}

Project supported by the National Natural Science Foundation of China (Grant Nos. 61072067 and 61372076), the 111 Project (Grant No. B08038), the Fund from the State Key Laboratory of Integrated Services Networks (Grant No. ISN 1001004), and the Fundamental Research Funds for the Central Universities (Grant Nos. K5051201022).

\section{REFERENCES}

[1] Masaki Akiyama, Masaki Koto, Shigenori Ioroi and Hiroshi Tanaka, "Implementation of a Smartphone-based Platform for the Operation of Home Electrical Appliances and Verification of its Feasibility," 2013 Seventh International Conference on Next Generation Mobile Apps, Services and Technologies, Prague, pp.38-43, Sept. 2013,

[2] Nomusa DLODLO, Andrew SMITH, Litsietsi MONTSI and Carel KRUGER, "Towards a Demand-Side Smart Domestic Electrical Energy Management System," IST-Africa Conference and Exhibition (IST-Africa), Nairobi, pp.1-12, May. 2013. 
[3] Engenlstad P, Egeland G and Thanh D V, "Analysis of NAT-Based Internet Connectivity for Multi-Homed On-Demand Ad Hoc Networks," Communication Networks and Distributed Systems Modeling and Simulation, 2004.

[4] Huaqiang $\mathrm{Xu}$, Lei Ju and Chongxian Guo, "On-demand gateway broadcast scheme for connectiong mobile ad hoc networks to the Internet," International Conference on Smart Computing (SMARTCOMP), Hong Kong, China, Nov. 2014, pp.112-117.

[5] Lu Dianjie, Zhang Guijuan and Zheng Xiangwei, "Analysis on Secure Connectivity in Ad Hoc Networks," Scientia Sinica (Informationis), vol.45, no.1, Jan. 2015, pp.97-110.

[6] Sayali N. Mane, N. V. Mane and D. G. Khaimar, "Performance of mobile node between different MANET with Mobile IP", 2015 International Conference on Industrial Instrumentation and Control(ICIC), Maharashtra, India. Aug. 2015, pp.1662-1664

[7] Dapeng Cheng, Yingjie Song, Yanyan Mao and Xiangrong Wang, "LDDP: A location-based directed diffusion routing protocol for smart home sensor network". 2014 2nd International Conference on Systems and Informatics (ICSAI 2014), Shanghai, China. Nov. 2014, pp.510-514.

[8] Mohamad,O.A., Hameed,R,T. and Tapus,N, "Smart home security based on optional wireless sensor network routing protocols", 2015 7th International Conference on Electronics, Computers and Artificial Intelligence(ECAI), Bucharest, Romania. June. 2015, pp.17-22.

[9] Rashid,A., Hasan,O. and Saghar,K, "Formal analysis of a ZigBeebased routing protocol for smart grids using UPPAAL," 2015 12th International Conference on High-Capacity Optical Networks and Enabling/Emerging Technologies(HONET), Islamabad, Pakistan. Dec. 2015, pp.1-5.

[10] Farooq, H. and Low Tang Jung, "Multi metric on-demand ad-hoc routing protocol for wireless smart metering deployment," 2013 IEEE 11th Malaysia International Conference on Communications (MICC), Kuala Lumpur, Malaysia. Nov. 2013, pp.140-144.

[11] Le, Quan, Ngo-Quynh, Thu,Magedanz and Thomaz, "RPL-based multipath Routing Protocols for Internet of Things on Wireless Sensor NetWork," 2014 7th International Conference on Advanced Technologies for Communications, Hanoi, Oct. 2014, pp.424-429.

[12] Xi Chen, "A Multiple Constrained Routing Selection Based on Particle Swarm Optimizaion Algorithms with Immunity", Journal of Changsha Communications University, vol. 22, no.2 , pp.56-5, 2006.

[13] Limin Meng and Wenbo Song, Mobile ad hoc network protocols, Beijing: Posts and Telecom Press, 2012, pp.62-74

[14] William J. Stewart., Probability, Markov chains, queues and simulation: the mathematical basis of performamce modeling, Beijing : Beijing World Publishing Corporation, 2013, pp.78-91. 